\title{
Investigating Dark Energy with the CMB lensing
}

\section{Viviana Acquaviva* SISSA/ISAS}

E-mail: acqualsissa.it

The nature of dark energy has been tentatively described by several different models, from a Cosmological Constant to a dynamical quintessence field, to a modification of the Einstein-Hilbert formulation of gravity. What we actually know about dark energy is that it gives raise to the present cosmic acceleration and it cannot have been relevant before quite late redshifts, or equivalently on very large scales. We chose to use the B modes of CMB polarization in order to discriminate among models. In fact, while most astrophysical observations are only sensitive to the local universe, the B modes signal is imprinted mainly by gravitational lensing at redshift $\mathrm{z}$ 1 , allowing to set constraints on the dark energy dynamics at this epoch which would be difficult to reach with photometric observations.

$C M B$ and Physics of the Early Universe 20-22 April 2006

Ischia, Italy

${ }^{*}$ Speaker. 

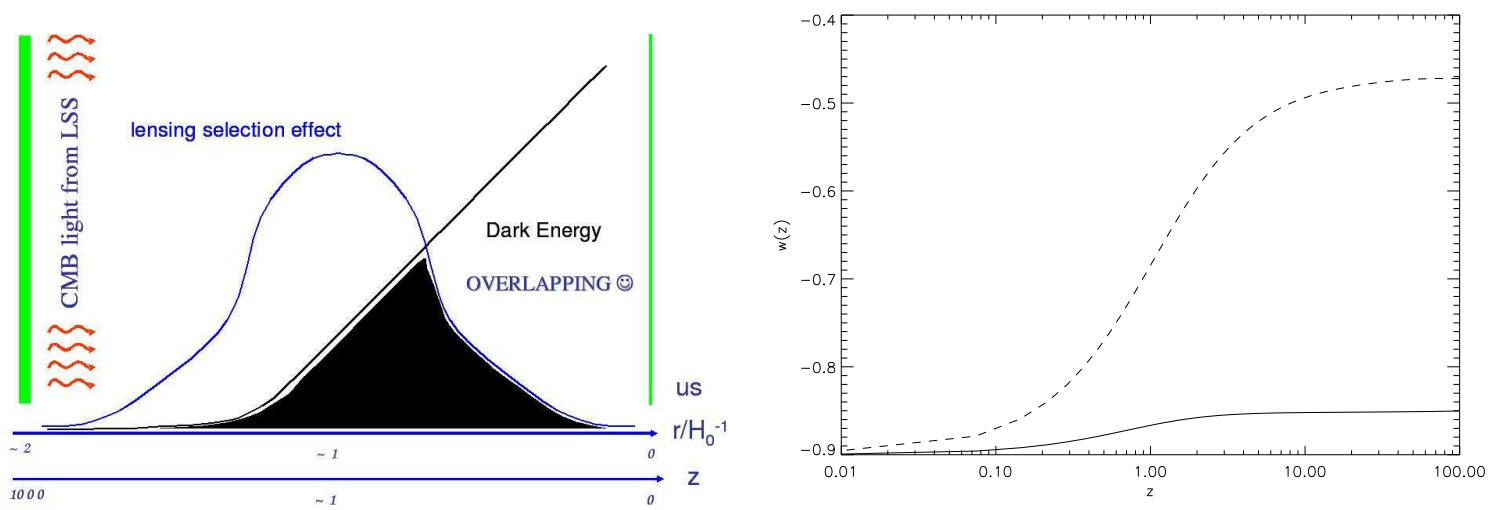

Figure 1: LEFT: The superposition between the dark energy contribution to the energy balance of the Universe and the lensing cross section. RIGHT: The equation of state of the dark energy for the SUGRA (dashed line) and IPL (solid line) models.

\section{Why CMB for dark energy?}

Gravitational lensing is a useful tool for the determination of cosmological parameters for at least two reasons: it correlates with the total mass distribution, with no regard for its interactions or state, and its cross section is largest at intermediate redshifts, where the models are allowed to most differ from each other. We show here a cartoon representation of how the lensing phenomenology "picks up" the signal at the most important epoch in the history of dark energy: it peaks somewhere in the middle between us and the last scattering surface, and this corresponds to a redshift window around $\mathrm{z}=1$. From the Fig. 1 one can also infer how the capability of this method will depend on the peculiar choice of the model: in fact, for earlier dark energy dominance the shaded area in the figure will be enhanced, and better constraints can be expected.

\section{CMB lensing phenomenology}

In order to understand how the lensing acts on the Cosmic Microwave Background spectra one needs to reconstruct how the observed lensed signal has been affected by its travelling through the matter distribution in its trip from the last scattering surface towards us. In order to do so, we have two groups of equations to solve: the Einstein equations, telling us how the matter (stress energy tensor) influences the structure of spacetime, and the lensing equation, which is the geodesic equation of motion giving the trajectores of photons in this modified spacetime. Once these equations have been solved, we find that that the lensed CMB fields can be expressed in the form of a Taylor series in powers of the deflection angle, which is the angle measuring the deviation in the photons trajectory caused by the gravitational lensing. The solution of the lensing equations ensures the reliability of this expansion, showing that the deflection angle is proportional to the (small) gravitational potential $\Psi$. Therefore, the lensed fields are written as

$$
X_{\text {lensed }}(\hat{\mathbf{n}})=X(\hat{\mathbf{n}}+\nabla \alpha)=X(\hat{\mathbf{n}})+\nabla_{i} \alpha \nabla^{i} X(\hat{\mathbf{n}})+O\left(\left(\nabla_{i} \alpha\right)^{2}\right) .
$$

The main implication of this equation is that lensing mixes power from different wavelengths, and, in the case of the $\mathrm{E}$ and $\mathrm{B}$ classification of polarization modes, from different types of perturbations 

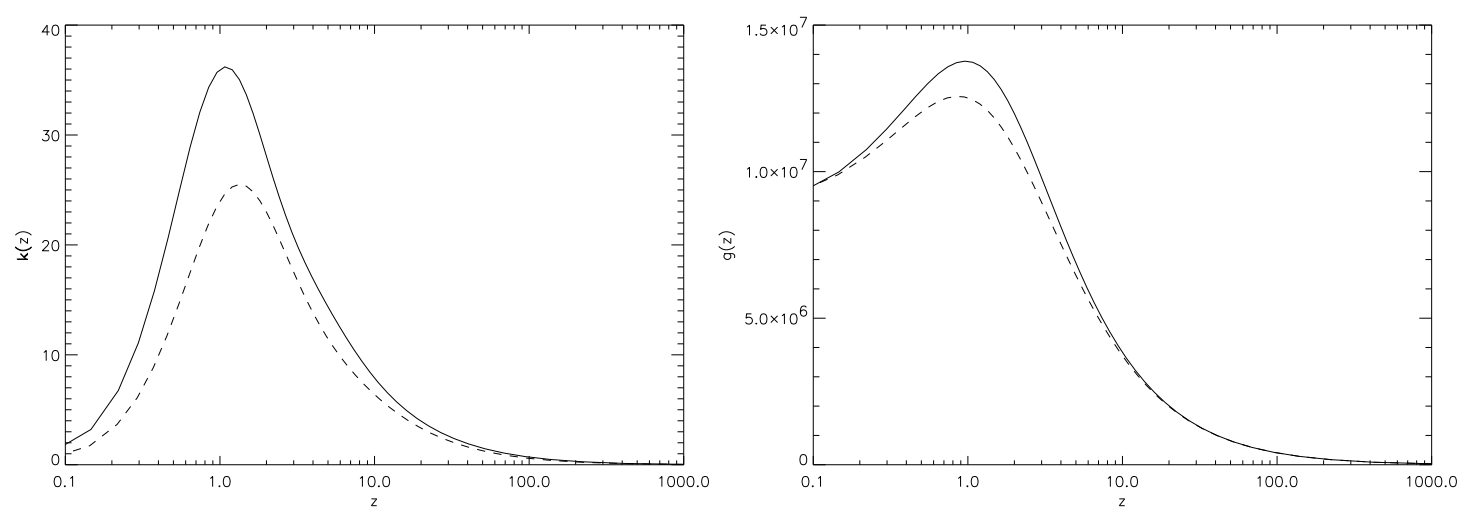

Figure 2: Lensing kernel (LEFT) and perturbation growth (RIGHT) at large scales for the SUGRA (dashed line) and IPL (solid line) models.

(scalar and tensor). Thus, although we can infer from the above equation that the first lensing correction is quadratic in the cosmological perturbations, where there is lack of primordial signal the lensing can be the unbiased responsible for the observed power: this is the case of the B modes for $1>100$.

\section{CMB spectra for Quintessence models}

We present here the spectra for two popular quintessence models, characterized by different potentials: one is an inverse power law (IPL), and the second is inspired by supergravity corrections (SUGRA). The models have been set to have the same equation of state of dark energy at present and the same amount of primordial perturbations, in order to highlight the difference in the spectradue to the different behaviour of the dark energy at intermediate redshifts. The lensed CMB spectra can be obtained through a convolution of the unlensed ones with a Gaussian function in the multipoles space [1] and receives two kinds of contributions: one from the different background evolution ("kernel") and one from the different perturbation growth. The equation of state, lensing kernel and growth function for a given wavenumber (notice we cannot separate any more the transfer function as $\left.T^{2}(k, z)=T^{2}(k, 0) g^{2}(z)\right)$ are respectively shown in Figs. 1 (right panel) and 2. Finally, we can show the temperature and B polarization spectra for the two models 3 . Notice that since the models have been set to have the same primordial and present behaviour, the large (almost 30\%) difference in the peak is entirely due to its sensitivity to the dark energy equation of state derivative. This is our most important result.

\section{A Fisher matrix analysis}

As a confirmation of the applicability of our method in view of the next generation of polarization experiments, we chose a parametrization for the dark energy equation of state as $w(z)=$ $w_{0}+\left(w_{\infty}-w_{0}\right)(1-a)$, using a standard set of other cosmological parameters. We performed [2] a Fisher matrix analysis on four models, a $\Lambda \mathrm{CDM}$, an IPL similar to the one above, and two (one 

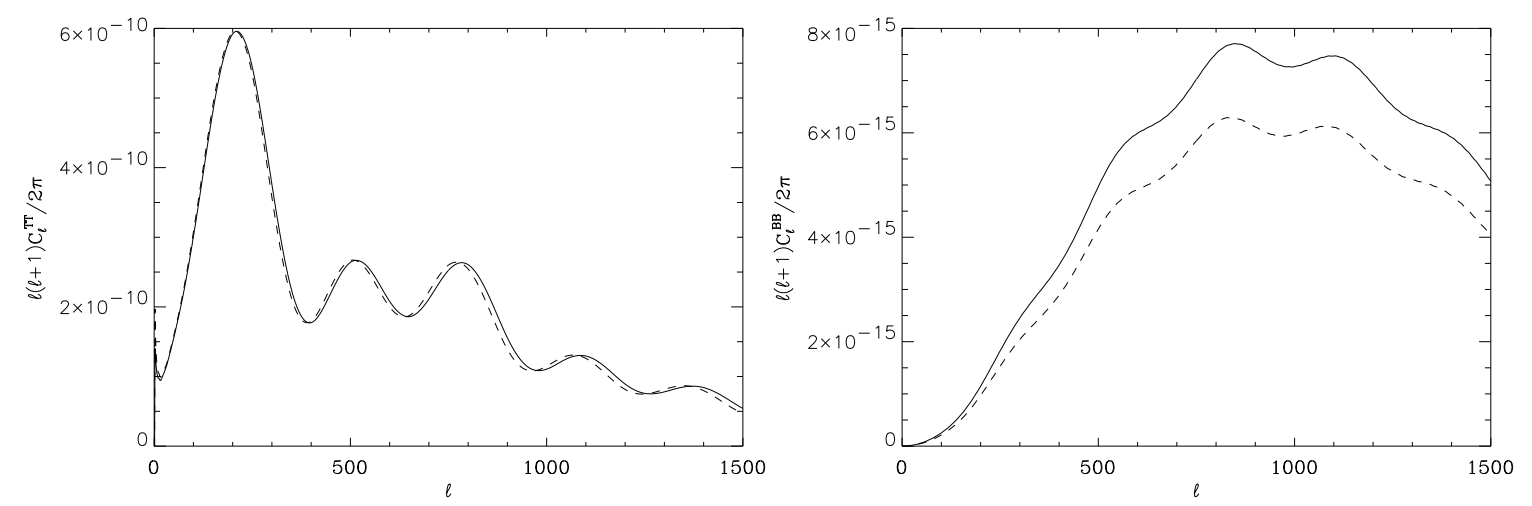

Figure 3: Temperature (LEFT) and B-polarization (RIGHT) spectra for the SUGRA (dashed line) and IPL (solid line) models.

"mild" and one "extreme") SUGRA models. Our target experiment is a CMBpol-like one in a quite conservative formulation.

\begin{tabular}{|lcc|cc|cc|cc|}
\hline & $\Lambda$ CDM & & IPL & & SUGRA1 & & SUGRA2 & \\
\hline & value & $\sigma_{\text {Fisher }}$ & value & $\sigma_{\text {Fisher }}$ & value & $\sigma_{\text {Fisher }}$ & value & $\sigma_{\text {Fisher }}$ \\
\hline$w_{0}$ & -1. & 0.12 & -0.9 & $9.7 \times 10^{-2}$ & -0.9 & $6.1 \times 10^{-2}$ & -0.82 & $3.5 \times 10^{-2}$ \\
$w_{\infty}$ & -1. & 0.27 & -0.8 & 0.19 & -0.4 & $6.9 \times 10^{-2}$ & -0.24 & $1.9 \times 10^{-2}$ \\
$\Omega_{b} h^{2}$ & 0.022 & $5.7 \times 10^{-5}$ & 0.022 & $6.0 \times 10^{-5}$ & 0.022 & $5.7 \times 10^{-5}$ & 0.022 & $5.9 \times 10^{-5}$ \\
$\Omega_{C} h^{2}$ & 0.12 & $7.0 \times 10^{-4}$ & 0.12 & $7.3 \times 10^{-4}$ & 0.12 & $6.6 \times 10^{-4}$ & 0.12 & $5.0 \times 10^{-4}$ \\
$h$ & 0.72 & $5.0 \times 10^{-2}$ & 0.72 & $4.5 \times 10^{-2}$ & 0.72 & $2.9 \times 10^{-2}$ & 0.72 & $1.5 \times 10^{-2}$ \\
$n_{S}$ & 0.96 & $2.1 \times 10^{-3}$ & 0.96 & $2.2 \times 10^{-3}$ & 0.96 & $2.1 \times 10^{-3}$ & 0.96 & $2.0 \times 10^{-3}$ \\
$\tau$ & 0.11 & $3.1 \times 10^{-3}$ & 0.11 & $3.0 \times 10^{-3}$ & 0.11 & $3.1 \times 10^{-3}$ & 0.11 & $3.2 \times 10^{-3}$ \\
$A$ & 1.0 & $5.6 \times 10^{-3}$ & 1.0 & $5.5 \times 10^{-3}$ & 1.0 & $5.5 \times 10^{-3}$ & 1.0 & $5.6 \times 10^{-3}$ \\
\hline
\end{tabular}

\section{Conclusions}

We have shown that our method of investigation through the B modes of polarization is especially sensitive to the dark energy equation of state at high redshift. We have developed the numerical procedure necessary to compute the lensed CMB spectra for a variety of dark energy models and presented quantitative results for the fiducial $\Lambda$ CDM model and three Quintessence ones. The Fisher matrix analysis showed that the next generation of polarization-devoted experiments will be capable of putting significant constraints, of the order between 10 and $20 \%$, on the dark energy equation of state derivative even for modestly dynamical models, and that the precision of the measurements increases with increasing dynamics.

\section{References}

[1] M. Zaldarriaga and U. Seljak, Phys. Rev. D 58, 023003 (1998).

[2] V. Acquaviva and C. Baccigalupi, astro-ph/0507644. 